\title{
Sir Francis Galton : le fondateur de l'eugénisme
}

> Explorateur, géographe de talent, météorologiste, biométricien, Sir Francis Galton est aussi le fondateur de l'eugénisme en 1883. L'eugénisme va se développer en se fondant sur une nouvelle théorie de l'hérédité, notamment exposée par Galton, mais aussi sur la théorie de l'évolution de Darwin, appliquée à la société humaine par Spencer. L'eugénisme de Galton est un programme de sélection artificielle pour produire une race humaine supérieure par un contrôle des mariages. Galton est favorable à un eugénisme positif espérant que les gens se fixeraient des objectifs eugénistes en choisissant leur partenaire en vue du mariage. En 1904, à Londres, il expose ses idées devant une foule de médecins et de scientifiques. Son discours, largement diffusé, servira de point de départ aux mouvements eugénistes américain et européen qui se développeront dans la première moitié du $X X^{e}$ siècle. $<$

Bien que J.P. Thomas affirme que "l'eugénisme fut autrefois une idéologie scientifique, il n'est plus aujourd'hui qu'une ânerie, dont on sourirait si sa persistance, bien au-delà du moment de sa destitution par la production progressive de connaissances nouvelles, n'avait donné lieu aux événements tragiques que l'on sait » [1], ce dernier est à nouveau au cœur des discours et des débats éthiques. Avec le développement des techniques de procréation médicalement assistée et la possibilité de diagnostiquer sur l'embryon ou le fœtus toutes sortes de maladies, on parle d'un «retour de l'eugénisme» [2] ou encore d'un «nouvel eugénisme» [3].

L'article 16-4 du Code civil aborde directement la question en interdisant les pratiques eugéniques tendant à la sélection des personnes [4], mais d'autres dispositions de la loi 94-654 autorisent, en les encadrant, certaines pratiques, comme le diagnostic préimplantatoire $[5,21]$, qui pourraient être qualifiées d'eugéniques. Le terme «eugénisme » provient étymologiquement de eu signifiant bien ou bon et de genos signifiant race ou
Dominique Aubert-Marson

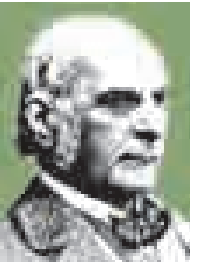

Maître de conférences, Laboratoire de biologie du développement et de la différenciation neuromusculaire, Université Paris Descartes, 45 , rue des Saints-Pères, 75006 Paris, France. aubert@univ-paris5.fr dominiquemarson@wanadoo.fr

naissance. Ce néologisme fut inventé par Francis Galton en 1883 mais l'idée eugénique s'était manifestée dans les sociétés antiques: chez les Hébreux, les Grecs et les Romains [6]. Par exemple à Sparte, un comité d'anciens examinait l'enfant nouveau-né et s'il le jugeait «mal venu et mal constitué, ils le faisaient jeter dans ce que l'on appelle les Aphotètes 》 [7].

Avec l'expansion du christianisme, l'élimination des enfants malformés disparaît, mais on retrouve les idées eugéniques à la Renaissance dans les cités utopiques décrites par More (Utopia, 1516) et Campanella (La Cité du soleil, 1623). À partir du XVII siècle, de nombreuses expressions vont désigner ce qui allait devenir l'eugénisme: callipédie (Quillet, 1655), gonocrotie (Millot, 1801), mégalanthropogénésie (Robert Le Jeune, 1803), aristogénie, orthopédie de la race, puériculture, viriculture... [8].

Après 1860 , les bases rationnelles de l'eugénisme sont posées: Spencer fonde le darwinisme social en appliquant la théorie de la sélection naturelle aux sociétés humaines, les méthodes statistiques se développent, les travaux de Mendel vont poser les fondements de la génétique, les caractères acquis sont rejetés par Weismann. D'autre part, l'urbanisation et la prolétarisation inhérentes à la révolution industrielle multiplient les maladies infectieuses, la pauvreté, la délinquance, l'alcoolisme, etc. La notion de dégénérescence prend une nouvelle ampleur avec la parution en 1857 du Traité des dégénérescences de Morel: ainsi, à cause de l'hérédité des maladies mentales, les dégénérés menacent la race entière. Deux autres œuvres qui s'intéressent au déclin des races ou des civilisations sont importantes au regard du développement de l'eugénisme: Une autre philosophie de l'histoire pour contribuer à l'éducation de l'humanité (1774) de Herder et Essai sur l'inégalité des races de Gobineau (1853-1855) qui sont tous deux hostiles au métissage, cause de la dégénérescence des nations [1].

\section{Francis Galton : apôtre de la quantification}

Francis Galton (1822-1911), né à Sparkbrook, près de Birmingham dans une vieille famille bourgeoise, est le cousin de Charles Darwin et 
aussi un parent de la famille Wedgwood, une famille d'industriels. Sa famille fonde de grands espoirs sur son avenir intellectuel car Galton sait lire à deux ans et demi ; à quatre ans, il sait écrire et compter ; à huit ans, il est à l'aise avec les textes latins classiques. Son père souhaitant qu'il devienne médecin comme son grand-père, il débute des études de médecine au King's College à Londres qu'il abandonne pour s'inscrire à l'université de Cambridge afin de suivre une licence de mathématiques. Préparant cette licence, il travaille beaucoup et est victime d'une dépression nerveuse - dont il aura des rechutes toute sa vie - déclenchée par le fait qu'il ne sort que second aux examens de mathématiques. Cette dépression joua un rôle important dans son choix de se diriger vers des secteurs où il n'aura pas ou peu de concurrents, comme l'hérédité et les statistiques [9]. Galton s'intéresse aux travaux de Quételet qui eut l'idée d'appliquer la théorie des probabilités et les méthodes statistiques aux sciences morales et politiques et aussi à l'anthropométrie, en inventant «l'hommemoyenne ». La statistique, sous toutes ses formes, fascine Galton qui trouve «cette science pleine de beauté et d'intérêt», c'est pour lui «le seul outil qui permette d'ouvrir une voie d'accès au sein du formidable foisonnement de difficultés barrant le chemin à ceux qui désirent faire l'étude scientifique de l'homme ».

En 1845, Galton part pour l'Égypte, la Syrie, l'Afrique du Sud. De ses voyages d'exploration il rapporte des observations qu'il traduit numériquement, mesure les «sauvages» et publie L'Art de voyager qui a obtenu une vaste audience [10]. Puis il se marie avec Louisa Butler mais ils n'auront pas d'enfant. C'est peutêtre pour cette raison qu'il s'intéresse à l'étude de l'hérédité et à l'eugénisme. II fait preuve d'une grande curiosité, parfois extravagante, et publie dans la revue Nature de nombreuses lettres comme par exemple «Comment couper

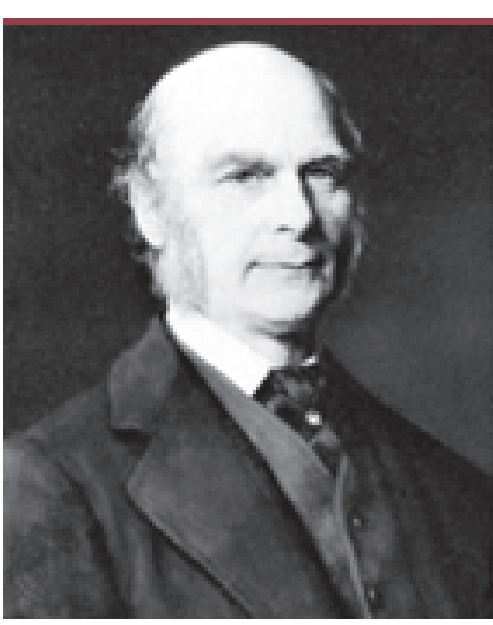

Sir Francis Galton (1822-1911) génie à l'état pur, un pionnier qui passait d'un sujet à l'autre, appliquant des méthodes mises au point dans un domaine pour résoudre un problème dans un autre secteur, souvent sans rigueur, et cependant généralement avec pertinence » [9].

\section{Le « génie héréditaire 》}

Si le contexte social joua un rôle important pour le développement de l'eugénisme, l'abandon du concept d'hérédité flexible ou «faible» (soft inheritance) jouera un rôle majeur. Cette hérédité stipule que les modifications acquises par les individus au cours de leur vie sont transmises à la génération suivante. À partir des années 1870, un nouveau concept de I'hérédité se répand: I'hérédité «inflexible»ou «forte» (strong inheritance) qui soutient que chaque organisme est constitué de deux parties: l'une est faite des caractères «patents» qui constituent le corps apparent, l'autre est constituée de caractères «latents» qui seront transmis aux descendants. Galton adhère à ce nouveau concept qui s'imposera vraiment, à partir de 1883, grâce à A. Weismann.

En 1868, Darwin énonce, comme une «hypothèse provisoire», sa théorie de la pangenèse. Cette théorie permet de concevoir la transmission héréditaire des variations selon un mode mécaniste. Darwin postule que les cellules de toutes les parties du corps émettent des «petits grains un cake pour le conserver trois jours », une carte de beauté des îles britanniques, etc. II est aussi un géographe de talent, membre de prestigieuses associations scientifiques dont la Société royale de géographie. On lui doit le terme d'«anticyclone» (1861) et de ses Meteographica (1863) il tire la première carte météorologique parue dans le Times du $1^{\text {er }}$ avril 1875.

Galton est qualifié par Gould d' «apôtre de la quantification» car il consacra son énergie et son intelligence à son passe-temps favori : la mensuration. Il fut un pionnier de la biométrie, statistiques appliquées à l'anthropométrie, et croyait que «la mensuration était le critère primordial de toute étude scientifique » [11]. Comme l'écrit Thuillier: «[...] Galton avait une tournure d'esprit essentiellement mathématique et statistique, il adorait compter; et Forrest (son biographe) lui-même suggère qu'il avait quelque chose d'obsessionnel dans ce goût du dénombrement et de la mesure » [12]. Sutter le présente comme "un savant trop méconnu dans notre pays, mais l'un des plus grands esprits du XIXe siècle » [13]. Forrest le qualifie de «génie victorien» [14]. Kevles le décrit comme étant «un ou atomes» nommés «gemmules» qui circulent dans le corps et s'agrègent «en éléments sexuels». Galton, voulant tester I'hypothèse de Darwin, étudia la descendance de lapins gris transfusés avec du sang de lapins blancs. Ce sang était supposé contenir des gemmules de lapins blancs, au bout de plusieurs générations les lapins gris devaient devenir blancs. Les résultats, publiés en 1871 dans un article intitulé « Experiments in Pangenesis by breeding from rabbits of a pure variety ", s'avérèrent négatifs, les lapins restaient blancs et il réfuta ainsi la théorie de Darwin qui protesta en affirmant que les gemmules n'étaient pas nécessairement véhiculées par le sang [15]. Galton opta alors pour une conception de l'hérédité proche de celle de Mendel dont les travaux restèrent inconnus jusqu'en 1900. En 1872, pour expliquer l'hérédité, Galton supposa l'existence de «stirps» (du latin stirp : racine), ensemble d'éléments responsables 
de la transmission des caractères d'une génération à l'autre: «Le stirp d'un enfant peut être considéré comme directement formé d'une partie des stirps de chacun de ses parents: dans ces conditions la structure personnelle de l'enfant n'est rien de plus qu'une représentation imparfaite de leurs propres stirps.» [16]

On lui doit également la plupart des idées sur l'hérédité de l'intelligence qui connurent un grand succès au début du XXe siècle avec le développement d'une école de la psychologie recourant aux tests, aux études de familles et à la statistique [11]. Galton établira ainsi que les qualités intellectuelles, comme les qualités physiques, sont héréditaires. Si l'environnement a une influence, tous les résultats lui donnent à penser que l'appartenance à une «race douée » (nature) joue un rôle majeur, l'environnement (nurture) jouant un rôle mineur. En 1865, Galton fait paraître un article dans la revue Macmillan's Magazine intitulé «Hereditary talent and character » où il expose l'analyse des biographies d'hommes célèbres en notant le nombre de parents éminents qu'elle possède. Il conclut son étude ainsi : "Je trouve que le talent est transmis par l'hérédité à un degré très remarquable. »

Puis il publie en 1869 Hereditary Genius, an inquiry into its laws and consequences dans lequel il analyse trois cents familles d'hommes éminents en tenant compte de diverses relations de parenté. Galton constate ainsi que $31 \%$ des hommes éminents avaient eux-mêmes un père éminent et que $38 \%$ ont un fils éminent. La conclusion qu'il tire de cette étude statistique est qu'il existe une loi de distribution des capacités dans les familles, c'est-à-dire que plus la parenté est proche, plus la proportion de génies est élevée.

Ses études chiffrées confirment l'existence d'inégalités entre les hommes qui ne connaîtront pas la même destinée sociale: certains réussissent, d'autres non. Galton assimile ainsi la distinction sociale au talent naturel: "Si un homme possède une grande valeur intellectuelle, s'il a le désir de travailler et la capacité de travailler voulue, je ne vois pas comment un tel homme pourrait être réprimé. »

Que Galton soit raciste est un fait incontestable. Dans ses écrits, le terme de race revient fréquemment mais il lui donne des sens différents. Ce terme de race renvoie à l'idée courante de race développée au XIXe siècle, c'est-à-dire race blanche, noire, jaune, etc. Mais Galton lui donne un second sens: la race désigne une classe sociale. Les différences entre les classes sociales, visibles par la réussite sociale, s'expliquent par des facteurs héréditaires. C'est la démarche initiale à partir de laquelle il construira l'eugénisme [1].
Il divise la «race anglaise » en trois classes sociales: I'aristocratie, à laquelle il s'identifie, qui correspond à l'élite et qu'il appelle les «désirables» (the gifted class), la bourgeoisie encore acceptable mais sans talent particulier et une classe importante de pauvres qu'il appelle les «indésirables ». Selon Galton et ses contemporains, la pauvreté correspond à un état biologique: le pauvre est pauvre car il est déterminé biologiquement ainsi. La stratégie eugénique est une stratégie réductionniste qui réduit les problèmes sociaux à des problèmes biologiques. Galton a peur de voir disparaître les élites au profit des pauvres dont le nombre augmente de génération en génération. Ainsi, l'eugénisme est la réponse d'une classe de la société apeurée face à l'angoisse du déclin ou de la dégénérescence et s'inscrit «dans le cadre d'une stratégie défensive » [1]. Galton s'intéressa aussi au système du français Alphonse Bertillon pour l'identification des criminels qui s'appuyait sur leurs mensurations physiques. Comme Cesare Lombroso dans L'Homme criminel (1876) chercha à décrire le «criminel-né » à travers l'étude de portraits photographiques de forçats montrant certaines caractéristiques anatomiques ou physiologiques [17].

\section{L’eugénique... une science?}

Galton écrit dans ses Mémoires: "Quand j'eus compris que l'hérédité des qualités mentales, sur lesquelles j'avais fait mes recherches, était réelle, et que l'hérédité était un moyen de développer des qualités humaines, beaucoup plus puissant que le milieu, je désirai explorer l'échelle des qualités dans des sens différents, en vue d'établir dans quelle mesure l'enfantement, tout au moins théoriquement, pouvait modifier la race humaine. Une nouvelle race pouvait être créée, possédant en moyenne un degré de qualité égal à celui rencontré seulement jusqu'ici dans les cas exceptionnels. » Pour créer cette «nouvelle race», Galton fonde l'eugénique. En 1883, il emploie dans Inquiries into Human Faculty pour la première fois le mot «eugénique » pour remplacer l'expression de «viriculture» (du latin vir, homme et cultura, culture) qu'il utilisait auparavant. Il définit ainsi l'eugénique: "science de l'amélioration de la race, qui ne se borne nullement aux questions d'unions judicieuses, mais qui, particulièrement dans le cas de l'homme, s'occupe de toutes les influences susceptibles de donner aux races les mieux douées un plus grand nombre de chances de prévaloir sur les races les moins bonnes ».

Le projet galtonien était de réorganiser la société anglaise en utilisant la sélection artificielle pour remplacer une sélection naturelle inefficace. Il constate que les méthodes utilisées par les éleveurs sont efficaces et permettent la reproduction d'animaux sélectionnés pour telle ou telle caractéristique. Pourquoi n'appliquerait-on pas à I'homme cette sélection artificielle afin de produire et de reproduire certains types mentaux comme le génie? II proclame sa conviction que «l'amélioration du cheptel humain ne posait aucune difficulté insurmontable ». II affirme dans Hereditary Genius qu'il est tout aussi facile «d'obtenir par une sélection attentive, les races de chiens et de chevaux dotés de qualités spéciales [...] et qu'il serait 
souhaitable de produire une race humaine supérieurement dotée par les moyens semblables ». Son objectif est avant tout national, et ne concerne que les «désirables», dont il craint la disparition, c'est-à-dire les ingénieurs, les médecins, les professions libérales, les militaires, les hommes d'État, etc. II faut davantage de bons scientifiques, de bons militaires, etc. pour renforcer la puissance de l'Empire anglais. Il est urgent d'«améliorer la race » car écrit-il: «Nous vivons dans une sorte d'anarchie intellectuelle par manque d'esprits supérieurs. En règle générale, la capacité intellectuelle de nos dirigeants a besoin d'être élevée. Nous voulons des capitaines, des hommes d'État, des penseurs et des artistes plus compétents. » Il est vrai qu'en Angleterre, les « désirables » ont un taux de fécondité plus faible que celui des «indésirables », d'où sa ferme volonté d'arrêter la dégénérescence en adoptant certaines mesures positives afin d'obtenir une élite plus nombreuse, comme un système d'examens et de bourses destiné à encourager la reproduction des élites. L'eugénisme sera donc «la clef d'un programme biologique visant la création d'une prudente méritocratie » [9].

Galton veut faire de l'eugénisme une religion «läque, substitut scientifique aux religions officielles» et prévoit «qu'une sorte de clergé scientifique prendrait le relais ». Donnant une dimension religieuse à l'eugénisme, il écrit dans The American Journal of Sociology (1905) : «L'eugénisme renforce le devoir social dans de si nombreuses circonstances que les conclusions résultant de son étude devraient recevoir un bon accueil de toute religion tolérante. [...] La foi eugéniste étend la philanthropie aux générations futures; elle rend son action plus pénétrante qu'elle ne l'a été jusqu'ici en prenant en considération les familles et les sociétés dans leur entièreté. [...] Elle interdit sévèrement toutes les formes de charité sentimentale qui sont nuisibles pour la race, en même temps qu'elle recherche activement les acres de bonté personnelle compensant la perte de ce qu'elle interdit. Elle attire l'attention sur les liens de l'espèce, et encourage fortement l'amour et l'intérêt pour la famille et la race. En bref, l'eugénisme est un credo viril, plein d'espoir, et faisant appel à nombre des nobles sentiments de la nature. »

Lors du VII Congrès international d'hygiène et de démographie, en 1891, qui se tient à Londres, Galton lance une Adresse aux démographes dont le thème central est la fécondité différentielle dans les diverses classes sociales au sein de l'Angleterre mais aussi des différentes fécondités parmi les nations elles-mêmes. Cette Adresse ne reçoit aucun succès, mais devant le peu d'enthousiasme des démographes, Galton ne se décourage pas et fonde plusieurs revues eugéniques comme Biometrika (1901) avec Weldon et Pearson, The Eugenics Review (1908) qui vont stimuler la création de sociétés eugéniques en Europe et aux États-Unis [18]. En 1904, Galton, pensant qu'il est temps d'agir, décide d'exposer ses idées devant la Société de sociologie de Londres dans une conférence-débat intitulée «L'eugénique, sa définition, son but, ses aspirations » où il propose un plan d'action visant à rassembler les connaissances sur l'hérédité, sur les taux de fécondité présents et passés des divers groupes sociaux, sur les influences qui ont déterminé les mariages au cours des âges, et ainsi faire de l'eugénique une discipline à part entière enseignée dans les universités afin de l'introduire «dans la conscience nationale comme une religion ». À cette conférence sont présents de nombreux médecins, les plus grands sociologues et de nombreux scientifiques. Cette conférence eut un vif succès, et sera imprimée et largement diffusée aux États-Unis et en Europe. C'est le point de départ du mouvement eugénique.

\section{Conclusion}

L'enthousiasme pour l'eugénique s'accroît dès le début $\mathrm{du} X X^{e}$ sècle et un nombre grandissant de personnes réclame l'adoption de mesures législatives. Les idées de Galton semblent toucher les hommes politiques et les premières législations rendant obligatoire la stérilisation des faibles d'esprit et des criminels furent votées dès 1907 aux États-Unis [19]. À la fin des années 1920, des lois semblables furent votées dans les pays scandinaves et en Allemagne nazie. Puis en octobre 1939, Hitler signa l'ordre de donner une «mort douce » aux malades incurables internés. Ainsi débuta l'opération T4 qui conduisit à la mort 275000 malades et vieillards [20]. Juste avant sa mort, Galton écrit dans Essays in eugenics (1909): «Il est avant tout nécessaire, pour que les progrès de l'eugénisme soient couronnés de succès, que ses défenseurs procèdent avec discrétion et ne prétendent pas à une efficacité plus grande que celle que le futur pourrait confirmer ». Le futur confirmera... Les politiques eugénistes mises en place n'eurent aucune efficacité sur le plan biologique et ne servirent qu'à porter atteinte à la dignité humaine. $\diamond$

\section{SUMMARY}

Sir Francis Galton: the father of eugenics

Not only was Sir Francis Galton a famous geographer and statistician, he also invented "eugenics" in 1883. Eugenics, defined as the scienceof improving racial stock, was developed from a new heredity theory, conceived by Galton himself, and from the evolution theory of CharlesDarwin, transposed to human society by Herbert Spencer. Galton'seugenics was a program to artificially produce a better human racethrough regulating marriage and thus procreation. Galton putparticular emphasis on "positive eugenics", aimed at encouraging thephysically and mentally superior members of the population to choosepartners with similar traits. In 1904, he presented his ideas in frontof a vast audience of physicians and scientists in London. Hiswidely-publicized lecture served as the starting point for thedevelopment of eugenics groups in Europe and the United States duringthe first half of the 20 th century. $\diamond$ 


\section{POUR EN SAVOIR PLUS}

L'ensemble de l'œuvre de Francis Galton est disponible en version numérisée en ligne sur le site:

http://www.galton.org

\section{RÉFÉRENCES}

1. Thomas JP. Les fondements de l'eugénisme. Collection Que sais-je? Paris: PUF, 1995 : 128 p.

2. Duster T. Retour à l'eugénisme. Paris : Kimé, 1992.

3. Gayon J, Jacobi D (eds). L'éternel retour de l'eugénisme. Collection Science, histoire et société. Paris : PUF, 2006.

4. Article 16-4 du Code civil : «Nul ne peut porter atteinte à l'intégrité de l'espèce humaine. Toute pratique eugénique tendant à l'organisation de la sélection des personnes est interdite ».

5. Article L 2131-4 du Code de santé publique.

6. Bresnu PN. L'eugénisme : théorie biologique et pratiques politiques. Université de Paris-Nanterre : Mémoire de DEA, 1985.

7. Plutarque. La Vie des hommes célèbres (La vie de Lycurgue). Collection La Pléiade. Paris : Gallimard, 1937.
8. Testart J. Le désir du gène. Paris : Flammarion, 1994.

9. Kevles DJ. Au nom de l'eugénisme. Paris: PUF, 1995.

10. Gillham NW. A life of Sir Francis Galton: from African explorer to the birth of eugenics. Oxford: Oxford University Press, 2001.

11. Gould SJ. La mal-mesure de l'homme, l'intelligence sous la toise des savants. Collection Biblio. Paris : Le Livre de Poche, 1986.

12. Thuillier P. Galton, un grand bourgeois de la science. La Recherche $1975 ; 56: 488-91$.

13. Sutter J. L'eugénique : problèmes, méthodes, résultats. Paris : PUF, 1950.

14. Forrest DW. Galton: the life and work of a Victorian genius. London : Paul Eleck, 1974.

15. Pichot A. La notion de gène. Collection Champs. Paris: Flammarion, 1999.

16. Galton F. A theory of heredity. Contemporary Review $1875 ; 27: 80-95$. Traduction française : Galton F. Théorie de l'hérédité. La Revue Scientifique 1876; 17 : 198-205.

17. Galton F. Composite portraits. J Anthropol Inst 1879; VIII : 132-44.

18. Pichot A. La société pure, de Darwin à Hitler. Paris: Flammarion, 2000.

19. Aubert-Marson D. Les politiques eugénistes aux États-Unis dans la première moitié du XXe siècle. Med Sci (Paris) $2005 ; 3: 320$-3.

20. Kogon $\varepsilon$, Langbein H, Rückerl A. Les chambres à gaz, secret d'État. Collection Points Histoire. Paris : Seuil, 1884.

21. Steffann J, Feyereisen $\varepsilon$, Kerbrat V, et al. Diagnostic prénatal et diagnostic préimplantatoire : arbre décisionnel, nouvelles pratiques? Med Sci (Paris) $2005 ; 21: 987-92$.

TIRÉS À PART

D. Aubert-Marson

\section{Prix Allergan de la SF0 2009}

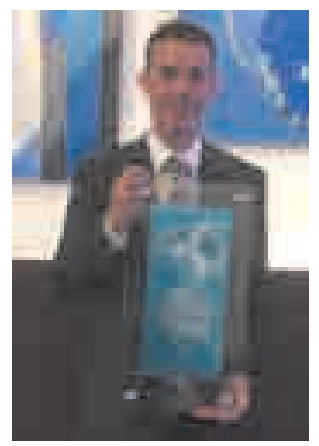

Docteur Cédric Lamirel lauréat du Prix 2009

Les laboratoires Allergan ont le plaisir de vous annoncer que le Prix Allergan de la SFO 2008 a été attribué cette année au Dr Cédric Lamirel (CHU d’Angers) pour un travail original intitulé «Une vision du glaucome depuis l'aire corticale V5/hMT». Ce prix a été décerné pendant le congrès de la SFO, le mardi 12 mai 2009.

Le Prix Allergan de la SFO récompense, à hauteur de $5000 €$, un travail de recherche original pharmacologique, clinique, paraclinique ou thérapeutique réalisé par un ophtalmologiste dans le domaine du glaucome.

Comité scientifique 2009: Professeurs Jean-Paul Renard (Hôpital Militaire du Val de Grâce - Paris), Jean-Philippe Nordmann (CHNO des Quinze-Vingts - Paris), JeanFrançois Rouland (Hôpital Huriez - CHRU de Lille), Philippe Denis (Hôpital Édouard Herriot - Lyon), Docteurs Éric Sellem (Centre Ophtalmologique Kléber - Lyon) et Philippe Lassalle (Laboratoire Allergan) sous la présidence du Dr Béatrice Cochener (CHU de Brest), présidente de la Société Française d’Ophtalmologie.

Les laboratoires Allergan renouvellent ce Prix pour l'année 2010, qui sera remis pendant le $116^{\mathrm{e}}$ Congrès de la SFO, en mai 2010. Les candidats devront soumettre leur dossier avant le $1^{\mathrm{er}}$ mars 2010.

Pour tout renseignement complémentaire sur les modalités de candidature, merci de vous adresser directement au secrétariat du Prix au 0492924476 ou à l'adresse Email suivante : lassalle_philippe@allergan.com 


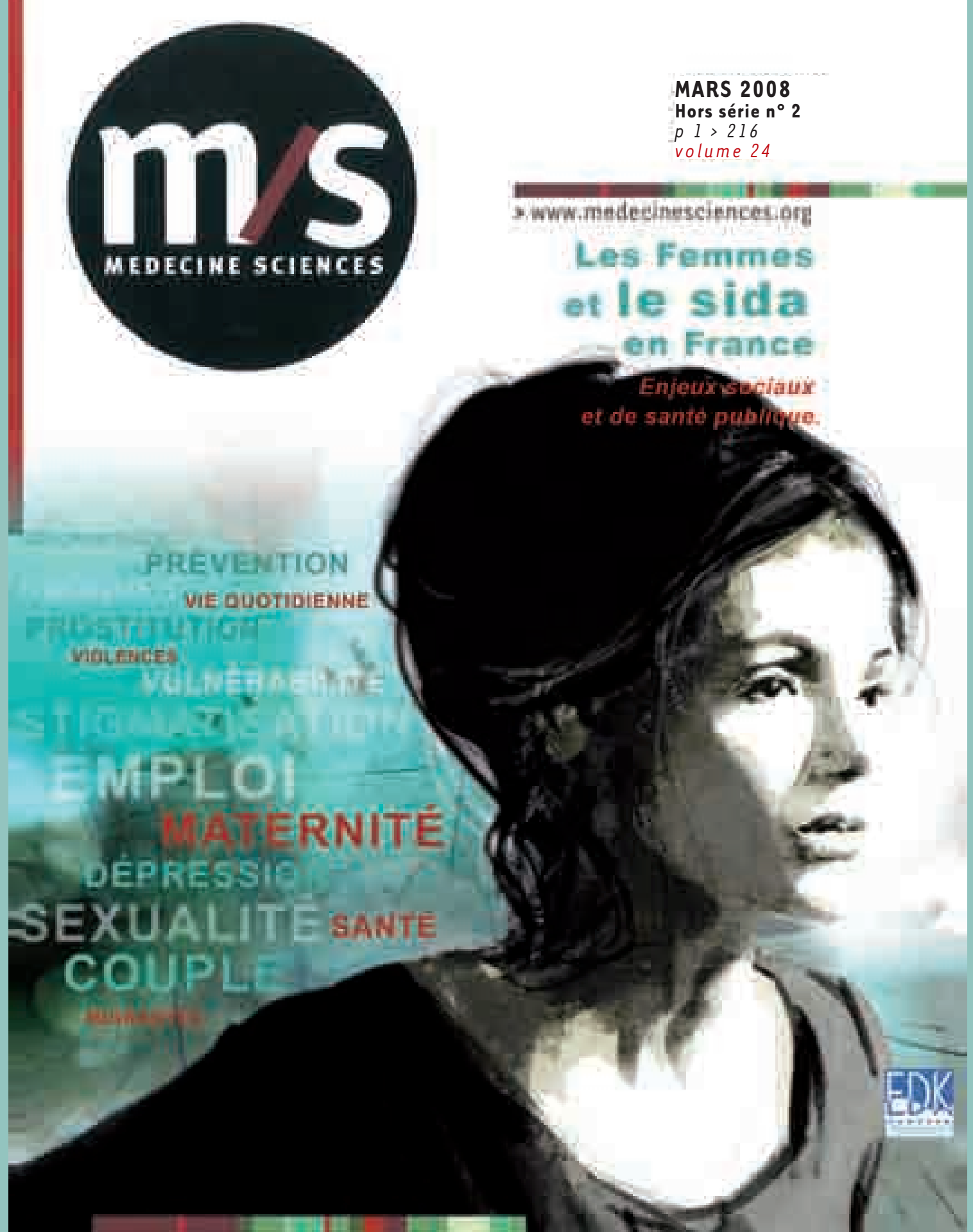

L’Anrs (Agence nationale de recherches sur le sida et les hépatites virales) publie « Les femmes et le sida en France - Enjeux sociaux et de santé publique ». Un numéro indispensable pour comprendre les nouveaux enjeux de prévention dans le contexte de féminisation et de précarisation de l'épidémie et au moment où se pose la question des conditions de vie des femmes atteintes par l'infection du VIH en France.

Un ouvrage précieux, destiné aux chercheurs, élus, décideurs en santé et représentants associatifs.

\section{Bon de commande}

À retourner à EDK, 2, rue Troyon - 92316 Sèvres Cedex

Tél. : 0155641393 - Fax : 0155641394 - E-mail : edk@edk.fr

NOM : Prénom :

Adresse :

Code postal :

Ville :

Pays :

Fonction :

Je souhaite recevoir le hors série ${ }^{\circ} 2$ de $M / S$ Les femmes et le Sida en France $: 18 €+3 €$ de port $=\mathbf{2 1} €$ TTC

en .............. exemplaire, soit un total de

$\square$ Par chèque, à l'ordre de $\mathbf{E} \mathbf{D} \mathbf{K}$

$\square$ Par carte bancaire : $\quad \square$ Visa $\square$ Eurocard/Mastercard

Carte $n^{\circ} \mid$\begin{tabular}{l|lllllllllllllllll}
$\mid$ & $\mid$ & $\mid$ & $\mid$ & $\mid$ & $\mid$ & $\mid$ & $\mid$ & $\mid$
\end{tabular}

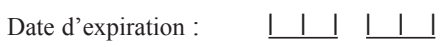

$\mathrm{N}^{\circ}$ de contrôle au dos de la carte : $\quad$ । । 